\title{
THE USE OF THE CONTRACTION MAPPING THEOREM WITH DERIVATIVES IN A BANACH SPACE*
}

\author{
By J. M. HOLTZMAN, Bell Telephone Laboratories, Incorporated \\ Whippany, New Jersey
}

I. Introduction. In order to use the contraction mapping fixed point theorem it is necessary to find a set mapped into itself such that the mapping is a contraction on that set. Because of the difficulty in finding the appropriate set mapped into itself, the contraction map theorem has been used mostly in cases where the mapping is a global contraction. Since this can impose severe restrictions, some authors have used the idea of simultaneously trying to determine a set mapped into itself and a contraction constant for that set. We present here a convenient formalization of this idea using derivatives in a Banach space. An application given in the present paper is to the existence of a subharmonic solution to a differential equation.

II. The contraction mapping theorem. The following is proven in $[1$, p. 661].

If $X$ is a Banach space and $P$ maps a convex closed subset $\Omega$ of $X$ into itself and if $P$ has a derivative at every point of $\Omega$, then

$$
\sup _{x \in \Omega}\left\|P^{\prime}(x)\right\|=\alpha<1
$$

implies that $P$ is a contraction on $\Omega$ (and thus there is a unique fixed point of $P$ in $\Omega$ ).

The following simple theorem is a help in applying the above result. that

Theorem. Let $B$ be a Banach space. $F$ maps $B$ into itself and $x_{0} \in B$. It is assumed

(i) F has a derivative at all $x \in B$.

(ii) There is a nondecreasing function $g$ such that if $x \in B$, then

$$
\left\|F^{\prime}(x)\right\| \leq g\left(\left\|x-x_{0}\right\|\right) .
$$

(iii) There is an $\alpha \in[0,1)$ such that

$$
g(k /(1-\alpha)) \leq \alpha
$$

where

$$
k \geq\left\|F\left(x_{0}\right)-x_{0}\right\| .
$$

Then there is a unique $x^{*} \in \Omega$ such that

$$
x^{*}=F\left(x^{*}\right)
$$

where

$$
\Omega=\left\{x: x \in B,\left\|x-x_{0}\right\| \leq k /(1-\alpha)\right\} .
$$

Proof. It is easily shown that $\left\|F^{\prime}(x)\right\| \leq \alpha$ for all $x \in \Omega$ and that $F$ maps $\Omega$ into itself. Thus, using the above quoted result from [1], there is a unique fixed point in $\Omega$.

${ }^{*}$ Received September 1, 1967; revised manuscript received November 3, 1967. 
Remarks. (i) See [1] for further discussion of fixed point theorems and differentiation in Banach spaces.

(ii) This result is less general than the version of the contraction mapping theorem given, e.g., on pp. 28, 29 of [2], but it seems simpler to apply when applicable. It is often a simple matter to find an appropriate function $g$.

(iii) It may sometimes be useful to obtain a bound on the first derivative by using the second Fréchet derivative. The appropriate condition is

$$
k_{2}+k_{3} k_{1} /(1-\alpha) \leq \alpha, \quad \alpha \in[0,1)
$$

with $\left\|F\left(x_{0}\right)-x_{0}\right\| \leq k_{1},\left\|F^{\prime}\left(x_{0}\right)\right\| \leq k_{2}$ and

$$
\left\|F^{\prime \prime}(x)\right\| \leq k_{3}, \quad x \in \Omega .
$$

III. The existence of a subharmonic solution to Duffing's equation. Our modification of the contraction mapping theorem is admittedly very simple. However, because of its simplicity, it seems to be useful in obtaining quick answers to some interesting problems. As an illustration, we shall consider the existence of a subharmonic solution to a differential equation. We get an explicit bound on the difference between an approximate and the exact solution.

Harmonic balance has indicated and experiment has verified the existence of a $\frac{1}{3}$ subharmonic solution to Duffing's equation

$$
\ddot{y}+a y+b y^{3}=f \cos 3 \omega t \quad(a, b, f>0) .
$$

It may be shown that under certain conditions (which are given on pp. 103-107 of [3]) that

$$
y(t)=A_{1} \cos \omega t+A_{3} \cos 3 \omega t
$$

satisfies Equation (III-1) if harmonics above the third are neglected.

For our analysis, we shall work in the Banach space of real-valued 2-vectors continuous on $[0, T]$ with norm

$$
\|x\|=\max _{i=1,2} \max _{t \in[0, T\}}\left|x_{i}(t)\right| .
$$

Letting $x_{1}=y, x_{2}=\dot{x}_{1}$, it is easily shown (see [4, p. 237]) that a continuous periodic solution of period $T=2 \pi / \omega$ to Equation (III-1) satisfies ${ }^{1}$

$$
x(t)=\int_{0}^{T} G(t, s)\left(\begin{array}{c}
0 \\
f \cos 3 \omega s-b x_{1}(s)
\end{array}\right) d s, \quad t \in[0, T]
$$

where

$$
G(t, s)=\frac{1}{2 \sin \left(\frac{\sqrt{a} T}{2}\right)}\left[\begin{array}{cc}
\sin \sqrt{a}\left(\frac{T}{2}-t+s\right) & \frac{1}{\sqrt{a}} \cos \sqrt{a}\left(\frac{T}{2}-t+s\right) \\
-\sqrt{a} \cos \sqrt{a}\left(\frac{T}{2}-t+s\right) & \sin \sqrt{a}\left(\frac{T}{2}-t+s\right)
\end{array}\right]
$$

It is assumed that $\ddot{y}+a y=0$ has no non-trivial periodic solution of period $T$. (Also, note that it is not actually necessary to convert to a vector integral equation.) 


$$
=\frac{1}{2 \sin \left(\frac{\sqrt{a} T}{2}\right)}\left[\begin{array}{cc}
-\sin \sqrt{a}\left(\frac{T}{2}+t-s\right) & \frac{1}{\sqrt{a}} \cos \sqrt{a}\left(\frac{T}{2}+t-s\right) \\
-\sqrt{a} \cos \sqrt{a}\left(\frac{T}{2}+t-s\right) & -\sin \sqrt{a}\left(\frac{T}{2}+t-s\right)
\end{array}\right]
$$

The integral on the right-hand side of (III-4) defines an operation $y=L N(x)$ where $L$ is defined by the linear integral operation. We are thus interested in fixed points of $L N(x)$. The method of harmonic balance as used in [3] finds an approximate solution $x_{0}$ satisfying

$$
x_{0}=L N\left(x_{0}\right)
$$

where $L$ is defined as follows:

$$
\begin{aligned}
& L N(x)(t) \sim \frac{a_{0}}{2}+\sum_{k=1}^{\infty}\left(a_{k} \cos k \frac{2 \pi}{T} t+b_{k} \sin k \frac{2 \pi}{T} t\right), \\
& L N(x)(t)=\sum_{k=1,3} a_{k} \cos k \frac{2 \pi}{T} t+b_{k} \sin k \frac{2 \pi}{T} t .
\end{aligned}
$$

Given an $x_{0}$ satisfying (III-6), we wish to determine if there is an $x^{*}$ satisfying

$$
x^{*}=L N\left(x^{*}\right)
$$

and if there is, how are $x_{0}$ and $x^{*}$ related? To apply our modification of the contraction mapping theorem, the Fréchet derivative operation $z=(L N)^{\prime}\left(x_{a}\right) x_{b}=L N^{\prime}\left(x_{a}\right) x_{b}$ is first obtained:

$$
z(t)=\int_{0}^{T} G(t, s)\left[\begin{array}{c}
0 \\
-3 b x_{a 1}^{2}(s) x_{b 1}(s)
\end{array}\right) d s .
$$

It is then easily shown that our modification of the contraction mapping theorem leads to seeking an $\alpha \in[0,1)$ satisfying

$3 b C T\left(\left|A_{1}\right|+\left|A_{3}\right|+\frac{C T b\left[\frac{3}{4}\left|A_{1}^{2} A_{3}+A_{1} A_{3}^{2}\right|+\frac{3}{4}\left|A_{1} A_{3}^{2}\right|+\frac{1}{4}\left|A_{3}^{3}\right|\right]}{1-\alpha}\right)^{2} \leq \alpha$

where

$$
C=\max \{1,1 / \sqrt{a}\} / 2|\sin (\sqrt{a} T / 2)| .
$$

The established existence of the fixed point near the approximation does not yet prove the existence of a subharmonic. However, this can be done as follows. Let $A_{1}^{*}$ be the coefficient of the cos $\omega t$ term in the Fourier series of $x_{1}^{*}(t)$ (which equals the sum of its Fourier series). If, $\max _{t}\left|x_{1}^{*}(t)-x_{01}(t)\right| \leq M$, then it easily follows that $\left|A_{1}^{*}-A_{1}\right| \leq$ $(4 / \pi) M$. Thus, $(4 / \pi) M<A_{1}$ implies that $A_{1}^{*} \neq 0$.

Let us consider the following numerical case (taken from [5, p. 65]):

$$
\begin{gathered}
a=2, \quad b=0.05, \quad f=2, \\
A_{1}=1.0, \quad A_{3}=-0.122, \quad \omega=4.28 \quad(T=1.47) .
\end{gathered}
$$

2 The $g$ function is $3 b C T\left(\max _{t}\left|x_{01}(t)\right|+\left\|x-x_{0}\right\|\right)^{2}$. 
For this case, $C=0.58$ and it is found that $\alpha=0.17$ satisfies (III-11) and further that

$$
\left\|x^{*}-x_{0}\right\| \leq 0.0039 /(1-0.17)=0.0047
$$

or

$$
\max _{t}\left|x_{1}^{*}(t)-x_{01}(t)\right| \leq 0.0047
$$

Conclusion. Problems of the type of Section III are discussed further in [6].

Acknowledgment. Helpful discussions with Prof. Hochstadt of Polytechnic Institute of Brooklyn are gratefully acknowledged. I also wish to thank I. W. Sandberg of Bell Labs for commenting on the draft.

\section{REFERENCES}

[1] L. V. Kantorovich and G. P. Akilov, Functional analysis in normed spaces, (translation), Pergamon Press, Oxford, England, 1964

[2] L. Liusternik and V. Sobolev, Elements of functional analysis, (translation), Ungar, New York, 1961

[3] J. J. Stoker, Nonlinear vibrations in mechanical and electric alsystems, Interscience, New York, 1950

[4] A. Halanay, Differential equations, Academic Press, New York, 1966

[5] N. W. McLachlan, Ordinary non-linear differential equations in engineering and physical sciences, 2nd Ed., Oxford at the Clarendon Press, 1956

[6] J. M. Holtzman, Contraction maps and equivalent linearization, Bell System Technical Journal (to appear, December 1967) 